\title{
Modelo populacional de Verhulst aplicado ao controle de potência distribuído DS/CDMA
}

\section{Verhulst populational model applied to distributed power control in DS/CDMA}

\author{
Tadeu Junior Gross ${ }^{1}$; Taufik Abrão²; Paul Jean Etienne Jeszensky ${ }^{3}$
}

\section{Resumo}

\begin{abstract}
Neste artigo, utiliza-se o modelo dinâmico contínuo de Verhulst, elaborado inicialmente para descrever o crescimento populacional de espécies biológicas com restrição de alimento e espaço físico, na síntese de um novo algoritmo de controle de potência distribuído (DPCA, Distributed Power Control Algorithm), aplicável em sistemas de múltiplo acesso por divisão de código (DS-CDMA, Direct Sequence-Code Division Multiple Access). Realiza-se a discretização da equação diferencial correspondente por meio do método de Euler de integração numérica (NIE, Numerical Integration Euler Method). São investigadas via simulações computacionais as propriedades do algoritmo recursivo assim obtido, como distância Euclidiana do vetor ótimo em regime, velocidade de convergência, erro quadrático médio normalizado (NSE, Mean Normalized Squared Error) na condição de regime, consumo médio de potência por usuário e complexidade de implementação. Os resultados são confrontados com resultados de simulações análogas para o algoritmo clássico de Foschini e Miljanic e o sigmoidal de Uykan e Koivo. Na presença de erros de estimação, o DPCA proposto apresentou menor discrepância em regime em relação ao vetor ótimo e maior velocidade de convergência. Adicionalmente, aplica-se a teoria dos Círculos de Gerschgorin (GC) na determinação de condições suficientes para que o problema de alocação de potência seja factível.

Palavras-chave: DS-CDMA. Algoritmo Controle de Potência Distribuído. DPCA. Modelo populacional de Verhulst. Círculos de Gerschgorin.
\end{abstract}

\begin{abstract}
In this paper the continuous dynamic model of Verhulst is used. It had been initially elaborated to describe the population growth of biological species with food restriction and physical space, in order to synthesize a new distributed power control algorithm (DPCA), applicable in direct sequence code division multiple access (DS-CDMA) systems. The discretization of the corresponding differential equation is accomplished via numeric integration Euler method (NIE). The properties of the proposed recursive algorithm, as Euclidian distance from optimum vector after convergence, convergence speed, normalized mean squared error (NSE), average power consumption per user and implementation complexity, are investigated through simulations. The simulation results are confronted with the other DPCAs: the classic algorithm derived by Foschini and Miljanic and the sigmoidal by Uykan and Koivo. With estimate errors, the proposed DPCA showed smaller discrepancy from the optimum power vector allocation after convergence and better convergence. Additionally, the Gerchgorin Circles theory (GC) is applied for the feasibility of the power allocation problem.
\end{abstract}

Key words: DS-CDMA. Distributed Power Control Algorithm. DPCA. Verhulst populational model.

\footnotetext{
1 Mestre em Engenharia Elétrica. Docente da Faculdade Unicen de Tangará da Serra - MT. E-mail: tj.gross@uol.com.br

2 Mestre e Doutor em Engenharia Elétrica. Desde 1997 é docente do Departamento de Engenharia Elétrica da Universidade Estadual de Londrina, PR Atualmente é professor adjunto AD-C. Suas áreas de interesse incluem comunicações sem fio, projeto de sequiências de códigos para uso em sistemas DS/CDMA, detecção multiusuário e estimação de parâmetros em DS/CDMA, técnicas heurísticas aplicáveis a problemas de otimização em sistemas de mútiplo acesso. E-mail: taufik@uel.br

3 Professor titular, Escola Politécnica da Universidade de São Paulo Departamento de Engenharia de Telecominicações e Controle, São Paulo-SP. E-mail:pjj@les.poli.usp.br
} 


\section{Introdução}

O trabalho de Foschini e Miljanic (FOSCHINI; MILJANIC, 1993) constitui o alicerce de diversos DPCA's difundidos na literatura, já que o processo de síntese desses DPCA's orienta-se no sentido de satisfazer à ODE (Ordinary Differential Equation) proposta em (FOSCHINI; MILJANIC, 1993), eq. (1). Além disso, verifica-se que a diretriz da maior parte das investigações existentes na literatura acerca de controle de potência distribuído tem sido o aprimoramento contínuo de estruturas de controle, atendo-se ao mesmo modelo dinâmico. Assim, a proposição de uma nova equação diferencial pode conduzir a algoritmos que sejam promissores sob algum aspecto significativo, tais como velocidade de convergência, proximidade do valor ótimo, sensibilidade a erros de estimação etc. Esse contexto justifica o novo modelo diferencial para síntese de DPCA's, baseado na equação de Verhulst (VERHULST, 1838). Este modelo é proposto e analisado aqui.

Para estruturação do problema de alocação, necessita-se de um modelo o mais próximo do cenário real. Primando pela generalidade, descreve-se a seguir um modelo multicelular, já que o unicelular pode ser visto como um caso particular. Assim, admitemse $K$ usuários ativos no canal reverso de um sistema DS-CDMA com $N$ células e considera-se modulação binária por chaveamento de fase, BPSK (Binary Phase Shifted Key).

O usuário $i$ (às vezes referenciado por $\mathrm{M}_{i}$ ) transmite seu sinal com potência $p_{i}$ e é demodulado na estação rádio base $\mathrm{B}_{i}$, onde chega com potência $a_{i i} p_{i}$, sendo $a_{i i}$ a atenuação provocada pelo canal de comunicação. De forma análoga, o sinal do usuário $j$ chega em $\mathrm{B}_{j}$ com potência $a_{j j} p_{j}$, conforme esquematizado na Figura 1. Adota-se que o critério de designação da estação rádio base é o da distância mínima, ou seja, cada usuário é demodulado por aquela que estiver mais próxima.

A potência interferente na entrada do receptor em $\mathrm{B}_{i}$, oriunda do sinal transmitido por $\mathrm{M}_{j}$, é dada por $a_{i j} p_{j}$. De modo similar, a interferência promovida por $\mathrm{M}_{i}$ em $\mathrm{B}_{j}$ vale $a_{j i} p_{i}$ (Figuras 1.a e 1.b). Cada usuário $\mathrm{M}_{i}$ é identificado por uma assinatura (seqüência de espalhamento). Sua forma de onda, denotada por $s_{i}(t)$, é não nula apenas no intervalo $\left[0, T_{b}\right]$ e possui energia unitária, isto é, $\int_{0}^{T_{b}} s_{i}^{2}(t) d t=1$. Supõe-se que a demodulação do sinal transmitido por $\mathrm{M}_{i}$ seja realizada em $\mathrm{B}_{i}$ por meio de um filtro casado a tal seqüência de espalhamento.

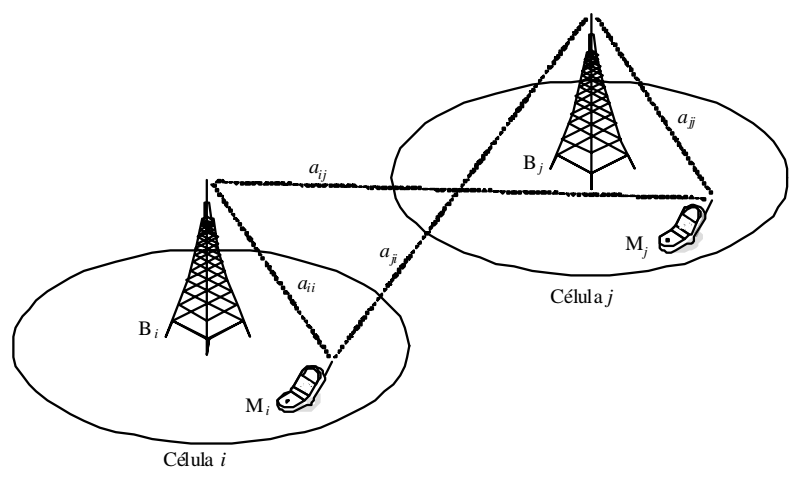

a

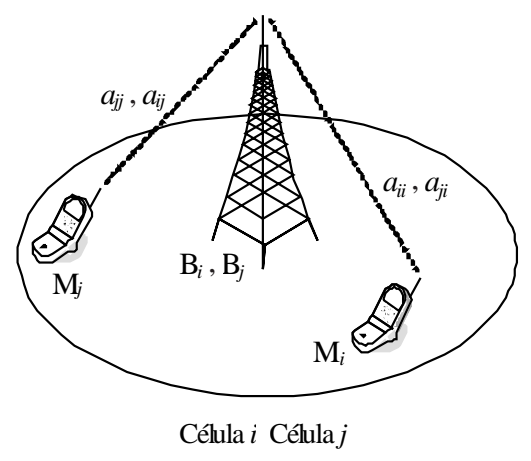

$\mathrm{b}$

Figura 1. Geometria para um sistema a) multicelular (considera-se usuários móveis localizados em células distintas) e b). unicelular (modelo considera apenas usuários móveis pertencentes a uma mesma célula).

Assume-se que o filtro casado referente ao usuário $\mathrm{M}_{i}$ esteja sincronizado com o atraso $\tau_{i i}$ Os demais usuários originam interferência assincronamente. Os atrasos relativos ao usuário $\mathrm{M}_{i}$, que podem possuir qualquer valor menor que a duração de um bit $\left(T_{b}\right)$, não sofrem variação temporal. No bit $\ell$ do usuário de interesse $\mathrm{M}_{i}$, outro usuário origina interferência por meio de seus bits $\ell$ 
e $(\ell-1)$ ou $\ell$ e $(\ell+1)$, dependendo do sinal do atraso relativo. Na Figura 2, as duas situações possíveis estão esquematizadas. $\mathrm{O}$ atraso do usuário $j$ relativo ao filtro casado do usuário $i$ é representado por $\tau_{i j^{*}}$ Ainda, o usuário $j$ possui um atraso positivo em relação ao usuário $i$ e cria interferência no bit $\ell$ de $i$ através de seus bits $(\ell-1)$ e $\ell$. Similarmente, o usuário $k$ tem um atraso relativo ao usuário $i$ negativo e cria interferência no bit $\ell$ de $i$ por meio de seus bits $\ell$ e $(\ell+1)$.

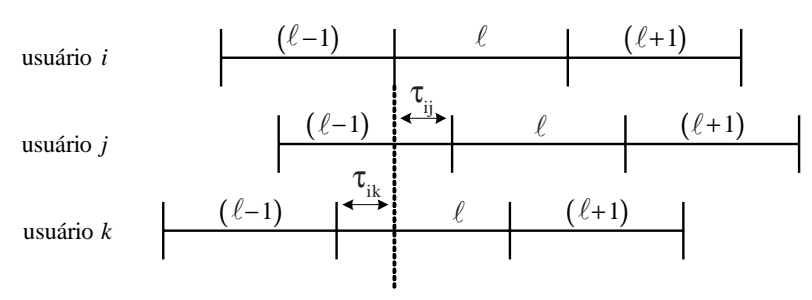

Figura 2. Desalinhamento temporal entre os usuários

Objetivando o cálculo da interferência de múltiplo acesso no bit $\ell$ do usuário $i$, definem-se três tipos de correlações cruzadas entre as assinaturas dos usuários $i$ e $j: \bar{\Gamma}_{i j}, \Gamma_{i j}$ e $\tilde{\Gamma}_{i j}$. Se $\tau_{i j} \geq 0$ (caso do usuário $j$ na Figura 2):

$$
\begin{aligned}
& \bar{\Gamma}_{i j}=\int_{0}^{\tau_{i j}} s_{i}(t) s_{j}\left(T_{b}-\tau_{i j}+t\right) d t, \\
& \Gamma_{i j}=\int_{\tau_{i j}}^{T_{b}} s_{i}(t) s_{j}\left(t-\tau_{i j}\right) d t e \\
& \tilde{\Gamma}_{i j}=0
\end{aligned}
$$

E se $\tau_{i j} \leq 0$ (caso do usuário $k$ na Figura 2):

$$
\begin{aligned}
& \bar{\Gamma}_{i j}=0 ; \quad \Gamma_{i j}=\int_{0}^{T_{b}+\tau_{i j}} s_{i}(t) s_{j}\left(t-\tau_{i j}\right) d t \quad e \\
& \tilde{\Gamma}_{i j}=\int_{T_{b}+\tau_{i j}}^{T_{b}} s_{i}(t) s_{j}\left(t-T_{b}-\tau_{i j}\right) d t
\end{aligned}
$$

Note-se que nos dois conjuntos de equações acima, $\bar{\Gamma}_{i j}$ ou $\tilde{\Gamma}_{i j}$ é igual a zero, implicando $\bar{\Gamma}_{i j} \tilde{\Gamma}_{i j}=0$ para todo $i, j$. Note também que $\Gamma_{i i}=1 \mathrm{e}$ $\bar{\Gamma}_{i i}=\tilde{\Gamma}_{i i}=0$.
A saída do $i$-ésimo filtro casado no bit $\lambda$ é:

$$
w_{i}(\ell)=\sum_{j=1}^{K} \sqrt{p_{j}} \sqrt{a_{i j}} \hat{b}_{i j}(\ell)+n_{i}(\ell)
$$

onde fez-se uso da definição:

$$
\hat{b}_{i j}(\ell)=\bar{\Gamma}_{i j} b_{j}(\ell-1)+\Gamma_{i j} b_{j}(\ell)+\tilde{\Gamma}_{i j} b_{j}(\ell+1)
$$

$\operatorname{com} b_{j}(\ell)$ o $\ell$-ésimo bit do usuário $j$ ( \pm 1 com igual probabilidade para modulação BPSK adotada aqui) e $n_{i}(\ell)$ é uma amostra do ruído branco aditivo Gaussiano, AWGN (Additive White Gaussian Noise), de média zero e variância $\sigma^{2}$. Observe-se que, embora $b_{j}(\ell)$ e $b_{j}(\ell+k)$ sejam independentes para $k \neq 0, \hat{b}_{i j}(\ell)$ e $\hat{b}_{i j}(\ell+k)$ não o são para $k= \pm 1$.

De (1) conclui-se que a interferência de múltiplo acesso é:

$$
\operatorname{MAI}_{i}(\ell)=\sum_{j=1, j \neq i}^{K} \sqrt{p_{j}} \sqrt{a_{i j}} \hat{b}_{i j}(\ell)
$$

Portanto, com variância:

$$
\sigma_{M A I_{i}}^{2}=\sum_{j=1, j \neq i}^{K} a_{i j} p_{j}\left(\bar{\Gamma}_{i j}^{2}+\Gamma_{i j}^{2}+\tilde{\Gamma}_{i j}^{2}\right)
$$

Dessa forma, a relação sinal-interferência mais ruído, SINR (Signal to Interference plus Noise Ratio) do usuário $i$, denotada por $\gamma_{i}$, é dada por:

$$
\gamma_{i}=\frac{a_{i i} p_{i}}{\sum_{j=1, j \neq i}^{K} a_{i j} p_{j}\left(\bar{\Gamma}_{i j}^{2}+\Gamma_{i j}^{2}+\tilde{\Gamma}_{i j}^{2}\right)+\sigma^{2}}
$$

De modo compacto:

$$
\begin{gathered}
\gamma_{i}=\frac{g_{i i} p_{i}}{\sum_{j=1, j \neq i}^{K} g_{i j} p_{j}+\sigma^{2},} \\
\operatorname{com} g_{i j}=a_{i j}\left(\bar{\Gamma}_{i j}^{2}+\Gamma_{i j}^{2}+\tilde{\Gamma}_{i j}^{2}\right) \text { e } g_{i i}=a_{i i}
\end{gathered}
$$

Tipicamente, pode-se definir distintas qualidade de serviços (QoS - Quality of Service) presentes 
no sistema em termos de probabilidade de erro de bit, que diminui com a SINR de forma monotônica. Desta forma, pode-se especificar QoS em termos de SINR.

Nas seções subsequentes, inicialmente, descrevese na seção II, de forma sucinta, o problema de alocação ótima de potência admitindo-se simplificação de sistema unicelular e deriva-se na seção III um método, à luz da Teoria dos Círculos de Gerschgorin (GC) (MEYER, 2000), que permite certificar de forma simples a existência do vetor ótimo. Na seção IV, apresentam-se duas metodologias distribuídas, o DPCA's clássico (FOSCHINI; MILJANIC, 1993) e o sigmoidal (UYKAN; KOIVO, 2004), visando à resolução do problema do controle de potência em sistemas de comunicação de múltiplo acesso. Na seção V, os autores propõem um novo DPCA baseado na ODE de Verhulst, enquanto na seção VI são apresentados resultados numéricos comparativos para os três algoritmos. Finalmente, na seção VII encontram-se sintetizadas as principais conclusões deste trabalho.

\section{O Problema de Alocação de Potência Ótima}

Em um sistema DS/CDMA unicelular com $K$ usuários móveis compartilhando o canal reverso, Figura 1.b, o sinal proveniente da unidade móvel $i$ será corretamente interpretado, com uma probabilidade de erro satisfatória, se a SINR na estação rádio-base, ERB (Estação Rádio Base) não for inferior a um determinado valor, denotado por $\gamma_{i}^{*}$ e estabelecido a priori. Portanto, deve-se controlar a potência transmitida de cada usuário móvel, $p_{k}$, com o intuito de obedecer a relação:

$$
\gamma_{i}=\frac{g_{i i} p_{i}}{\sum_{j=i, j \neq i}^{K} g_{i i} p_{j}+\sigma_{i}^{2}} \geq \gamma_{i}^{*}, i=1, \ldots, K
$$

onde $g_{i j}$ corresponde ao fator de atenuação do sinal do usuário interferente $j$ e $\sigma_{i}^{2}$ à potência do ruído AWGN inerente ao sistema de comunicação.
O vetor de potências que transforma as $K$ inequações acima em igualdades também minimiza a soma das potências transmitidas (ULUKUS; YATES, 1998). Portanto, ao impor-se esta restrição, tem-se $K$ equações que podem ser convenientemente agrupadas em um formato matricial:

$$
\begin{aligned}
& (\mathbf{I}-\mathbf{H}) \mathbf{p}=\boldsymbol{\eta}, \\
& \text { sendo } h_{i j}=\gamma_{i}^{*} g_{i j} g_{i i}^{-1}, h_{i i}=0 \\
& \text { e } \eta_{i}=\gamma_{i}^{*} \sigma_{i}^{2} g_{i i}^{-1}
\end{aligned}
$$

A síntese de um DPCA consiste em desenvolver um procedimento sistemático de evolução do vetor $\mathbf{p}$, baseando-se nos valores $\gamma_{i}^{*}, \gamma_{i}$ e $p_{i}$, para o vetor ótimo:

$$
\mathbf{p}^{*}=(\mathbf{I}-\mathbf{H})^{-1} \boldsymbol{\eta}
$$

A existência de uma solução não negativa para (3) segue de dois teoremas, atribuídos a Perron e Frobenius (SENETA, 1991), acerca de autovalores de matrizes não negativas.

Teorema 1: Se $S$ é uma matriz quadrada não negativa, existe um autovalor $\rho$, denominado autovalor de Perron-Frobenius, de forma que: 1) $\rho$ é real e não negativo; 2) $\rho \geq|\lambda|$ para qualquer autovalor $\lambda$ de $\boldsymbol{S}$. No plano complexo, o raio $\mathrm{r}(\mathbf{S})$ do menor círculo que contém todos os autovalores de uma matriz $\mathbf{S}$ qualquer, ou seja, $r(\mathbf{S})=\max \{|\lambda|: \lambda \in \lambda(\mathbf{S})\}$, denomina-se raio espectral da matriz $\mathbf{S}$ (MEYER, 2000). Portanto, para uma matriz quadrada não negativa, raio espectral e autovalor de PerronFrobenius podem ser utilizados sem distinção.

Teorema 2: Para uma matriz não negativa irredutível $\mathbf{S}$ e um vetor $\mathbf{c}$ estritamente positivo, $\mathbf{x}=$ $\mathbf{S x}+\mathbf{c}$ possui solução $\mathbf{x}$ não negativa se, e somente se, $o$ autovalor $\rho$ de Perron-Frobenius for menor que a unidade.

Da equação (3) e do Teorema 2, conclui-se que o sistema em questão é factível se o raio espectral da matriz $\mathbf{H}$ for $\rho_{\mathbf{H}}<1$. Portanto, torna-se necessário identificar quais condições os elementos da matriz 
H devem satisfazer de forma que os autovalores estejam limitados ao círculo unitário.

Uma maneira precisa de relacionar os elementos de $\mathbf{H}$ com seus autovalores consiste em analisar a influência dos coeficientes do polinômio característico sobre suas raízes. Este procedimento clássico tornase inadequado quando o grau do polinômio for $n>4$, pois, exceto em casos particulares, não existe solução fechada para encontrar zeros de tais polinômios. Neste contexto, métodos aproximados para localizar autovalores revelam-se importantes.

A Teoria dos GC (MEYER, 2000) permite a obtenção de um procedimento simples para se delimitar regiões do plano complexo que contêm autovalores. A utilização desta teoria, na derivação de condições suficientes para garantir viabilidade ao problema do controle de potência, constitui procedimento não explorado na literatura e será descrito a seguir.

\section{Sistema Factível e os Elementos da Matriz $\mathbf{H}$ à Luz da Teoria dos Círculos de Gerschgorin}

O Teorema de GC, aliado às características particulares da matriz $\mathbf{H}$, revela-se uma ferramenta prática na obtenção de condições suficientes para viabilidade de um sistema de controle de potência. $\mathrm{O}$ Teorema de GC pode ser enunciado como (MEYER, 2000):

Teorema 3: O espectro $\lambda(\Delta)$ de uma matriz $\Delta_{n \times n}$ está contido em uma união $G(\boldsymbol{\Delta})$ de círculos centrados nos elementos da diagonal principal. O raio $R_{i}(\Delta)$ da região circular $\mathscr{D}_{i}(\boldsymbol{\Delta})$ associada a $\delta_{i i}$ é obtido somando-se os módulos dos elementos pertencentes à linha $i$ que não estejam localizados na diagonal principal, ou seja:

$$
\begin{gathered}
R_{i}(\Delta)=\sum_{\ell=1, \ell \neq i}^{n}\left|\delta_{i \ell}\right| \\
\operatorname{com} \mathscr{D}_{i}(\boldsymbol{\Delta})=\left\{\mathrm{z} \in \mathcal{C}:\left|\mathrm{z}-\delta_{i i}\right| \leq R_{i}(\Delta)\right\}
\end{gathered}
$$

$$
\begin{gathered}
G(\boldsymbol{\Delta})=\bigcup_{i=1}^{n} \mathcal{D}_{i}(\boldsymbol{\Delta}) \\
\lambda(\boldsymbol{\Delta}) \subset G(\boldsymbol{\Delta})
\end{gathered}
$$

Os discos $\mathscr{D}_{i}(\boldsymbol{\Delta}), i=1, \ldots, n$, são denominados Círculos de Gerschgorin (GC) (MEYER, 2000) e o fato dos autovalores de $\Delta$ estarem localizados na união dessas regiões pode ser referenciado como Teorema de GC. Cada disco disjunto contém necessariamente um autovalor.

Os espectros de matrizes transpostas são idênticos, e, portanto, $\lambda(\boldsymbol{\Delta}) \subset G(\boldsymbol{\Delta}) \cap G\left(\boldsymbol{\Delta}^{\mathbf{T}}\right)$. Esta intersecção diminui a região de busca no plano complexo, melhorando a estimativa inicial sobre a localização dos autovalores.

Os elementos da diagonal principal da matriz $\mathbf{H}_{n \times n}$ são nulos por definição e, portanto, todos os GC são concêntricos e centrados na origem do plano complexo. Dessa forma, o disco $\mathscr{D}_{k}(\mathbf{H})$ de maior raio contém a união de todos os GC, ou seja, existe pelo menos um $k \in\{1, \ldots, n\}$ de modo que $G(\mathbf{H}) \subset \mathcal{D}_{k}(\mathbf{H})$. Conseqüentemente, para este índice $k$ vale a desigualdade $\mathrm{r}(\mathbf{H}) \leq R_{k}(\mathbf{H})$. Analogamente, como $\lambda(\mathbf{H})=\lambda\left(\mathbf{H}^{\mathrm{T}}\right)$, obtém-se $\mathrm{r}(\mathbf{H}) \leq R_{\lambda}\left(\mathbf{H}^{\mathrm{T}}\right)$ para algum $\lambda \in\{1, \ldots, n\}$. Agrupando-se estas duas desigualdades, obtém-se um limite mais estreito para o raio espectral, ou seja, $\mathrm{r}(\mathbf{H}) \leq \min \left\{R_{k}(\mathbf{H}), R_{\lambda}\left(\mathbf{H}^{\mathbf{T}}\right)\right\}$.

A não-negatividade da matriz quadrada $\mathbf{H}$ implica que seu raio espectral $r(\mathbf{H})$ e seu autovalor de Perron-Frobenius $\rho_{\mathrm{H}}$ são iguais e, portanto, $\rho_{\mathbf{H}} \leq \min \left\{R_{k}(\mathbf{H}), R_{\lambda}\left(\mathbf{H}^{\mathrm{T}}\right)\right\}$. Como um sistema factível deve necessariamente possuir $\rho_{\mathbf{H}}<1$, a restrição $\min \left\{R_{k}(\mathbf{H}), R_{\lambda}\left(\mathbf{H}^{\mathbf{T}}\right)\right\}<1$, onde $k$ identifica o maior disco da matriz $\mathbf{H}$ e $\lambda$ o maior de sua transposta, estabelece uma condição suficiente para viabilidade.

Sistematicamente, os passos para verificar se o problema de alocação de potências descrito por (3) tem solução ótima são: 
1- Calcular a soma dos elementos da linha de $\mathbf{H}$ que possuir maior norma:

$$
R_{\text {row }}^{\max }=\max _{k}\left\{\sum_{\ell=1}^{n} h_{k \ell}\right\}
$$

2- Calcular a soma dos elementos da coluna de $\mathbf{H}$ que possuir maior norma:

$$
R_{c o l}^{\max }=\max _{\ell}\left\{\sum_{k=1}^{n} h_{k \ell}\right\}
$$

3- Excluir o maior valor entre os dois anteriores:

$$
R_{G_{r} \cap G_{c}}^{\max }=\min \left\{R_{\text {row }}^{\max }, R_{c o l}^{\max }\right\}
$$

4- Valor resultante menor do que 1 implica viabilidade:

$$
\begin{gathered}
R_{G_{r} \cap G_{c}}^{\max }<1 \Rightarrow \text { sistema factível; } \\
R_{G_{r} \cap G_{c}}^{\max } \geq 1 \Rightarrow \text { nada se pode afirmar }
\end{gathered}
$$

A confirmação da viabilidade por esta metodologia simples descarta a necessidade da utilização de qualquer método numérico de cálculo do autovalor de Perron-Frobenius. Na situação em que $R_{G_{r} \cap G_{c}}^{\max } \geq 1$, não se pode garantir que o sistema é factível e a certificação da existência do vetor ótimo deve ser feita por outro mecanismo numérico. Neste caso, o quão maior $R_{G_{r}}^{\max } \cap$ for da unidade pode ser entendido como uma medida da incerteza que este método fornece sobre a viabilidade.

A condição suficiente para que os GC de $\mathbf{H}$ ou $\mathbf{H}^{\mathrm{T}}$ pertençam à região interna do círculo unitário permite verificar a tendência de viabilidade conforme se variam os parâmetros que definem $h_{i j}$.

Em um esquema DS-CDMA com receptor convencional, o desalinhamento temporal entre os usuários que compartilham o canal reverso impede o uso de seqüências de espalhamento ortogonais e, portanto, a SINR na saída de cada filtro casado depende das propriedades de correlação cruzada entre as sequiências utilizadas. De acordo com (2), a potência do usuário interferente $j$ é atenuada por $g_{i j}$. Esse ganho é tão menor quanto o for a correlação cruzada entre os usuários $i$ e $j$ (ULUKUS; YATES, 1998), eq. (6). Como $h_{i j} \propto g_{i j}$, baixas correlações cruzadas tendem a diminuir os GC e, portanto, tornar o sistema factível.

Analogamente, o ganho $g_{i j}$ é influenciado pelo canal de rádio móvel (ULUKUS; YATES, 1998, eq. (6). e, como $h_{i j} \propto g_{i j} g_{i i}{ }^{-1}$, ocorrem flutuações temporais no valor de $R_{G_{r} \cap G_{c}}^{\max }$. A amplitude do sinal transmitido por um determinado usuário sofre desvanecimento de larga escala, $m(t)$, e de pequena escala, $r(t)$ (SKLAR, 1997) e, dessa forma, tem-se $g_{i j} \propto \quad\left(m_{i j} r_{i j}\right)^{2}$ e $\quad h_{i j} \propto\left(\frac{m_{i j} r_{i j}}{m_{i i} r_{i i}}\right)^{2}$. Portanto, desvanecimento profundo sobre o sinal de interesse $i$ aliado à interferência construtiva dos multipercursos do usuário $j$ podem fazer com que $R_{G_{r} \cap G_{c}}^{\max }$ atinja valor elevado, indicando tendência à inviabilidade. Adicionalmente, se o usuário $i$ estiver mais próximo da ERB que o $j$, o fator $m_{i j} / m_{i i}$ diminui fazendo com que a incerteza sobre a viabilidade seja menor.

Finalmente, como $h_{i j} \propto \gamma_{i}^{*}$, altos níveis de SINR tendem a aumentar os GC, indicando que o sistema pode tornar-se não factível. Neste caso, relaxações adequadas para atender as distintas qualidade de serviços $(\mathrm{QoS})$ críticos viabilizam a implementação do controle de potência.

As análises relativas aos DPCA's expostos neste artigo pressupõem que o autovalor de PerronFrobenius da matriz $\mathbf{H}$ seja menor do que a unidade.

\section{Algoritmos de Controle de Potência Distribuídos}

A solução ótima do problema de alocação satisfaz o seguinte conjunto de equações (ver 3):

$$
p_{i}^{*}=\sum_{j \neq i}^{K} h_{i j} p_{j}^{*}+\eta_{i}, \quad i=1, \ldots, K
$$


Agora, considere uma função ímpar arbitrária $y_{i}=z_{i}(x)$ que tenha valor nulo apenas na origem. Assim, (13) constitui o único ponto de equilíbrio do seguinte sistema dinâmico de tempo contínuo:

$$
\dot{p}_{i}=z_{i}\left(-p_{i}+\sum_{j \neq i}^{K} h_{i j} p_{j}+\eta_{i}\right), i=1, \ldots, K
$$

onde $\dot{p}_{i}$ indica a primeira derivada temporal de $p_{i}$. Na Figura 3, estrutura-se o diagrama de blocos referente a (14).

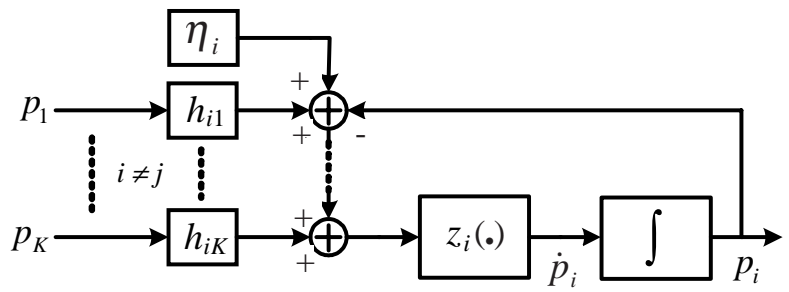

Figura 3. Sistema dinâmico com equilíbrio na solução do problema de alocação

A reta secante à $p_{i}(t)$ nos instantes $t$ e $\alpha_{i}+t$ é indistingüível da tangente em $t$ quando $\alpha_{i}$ for suficientemente pequeno, supondo que $p_{i}(t)$ seja uma curva suave. Desta forma, vale a aproximação:

$$
\begin{gathered}
p_{i}\left(t+\alpha_{i}\right) \approx p_{i}(t)+\alpha_{i} z_{i}\left(-p_{i}(t)+\sum_{j \neq i}^{K} h_{i j} p_{j}(t)+\eta_{i}\right), \\
\alpha_{i}>0, i=1, \ldots, K
\end{gathered}
$$

que consiste no conhecido método de Euler de integração numérica (PRESS et al., 1992).

Do exposto, a seqüência $p_{i}[n]=p_{i}\left(n \alpha_{i}\right), n \in \mathrm{N}$, pode ser obtida recursivamente por:

$$
\begin{gathered}
p_{i}[n+1]=p_{i}[n]+\alpha_{i} z_{i}\left(-p_{i}[n]+\sum_{j \neq i}^{K} h_{i j} p_{j}[n]+\eta_{i}\right), \\
i=1, \ldots, K
\end{gathered}
$$

Das definições em (2) e (3), verifica-se que: $-p_{i}+\sum_{j \neq i}^{K} h_{i j} p_{j}+\eta_{i}=-\left(1-\frac{\gamma_{i}^{*}}{\gamma_{i}}\right) p_{i}, \quad i=1, \ldots, K$

Portanto, (16) equivale a:

$p_{i}[n+1]=p_{i}[n]-\alpha_{i} z_{i}\left(\left(1-\frac{\gamma_{i}^{*}}{\gamma_{i}[n]}\right) p_{i}[n]\right), i=1, \ldots, K$

A recursão em (18) pode ser efetivamente implementada na unidade móvel $i$. Admite-se que o parâmetro $\alpha_{i}$, o nível de QoS quantificado por $\gamma_{i}^{*}$, a função $z_{i}(\bullet)$ e o valor da potência transmitida $p_{i}[n]$ estejam disponíveis na unidade móvel $i\left(\mathrm{M}_{i}\right.$ na Figura 4). Entretanto, a SINR $\gamma_{i}[n]$ pode ser obtida somente na estação rádio base $\mathrm{B}_{i}$, aquela que demodula $\mathrm{o}$ sinal do usuário $i$. Dessa forma, para que o usuário $i$ calcule por meio de (18) o próximo nível de potência, $\mathrm{B}_{i}$ precisa estimar $\gamma_{i}[n]$, quantizar o resultado em um número conveniente de bits e, em seguida, realimentá-lo para o usuário $i$ através do canal direto. Todo o processo está esquematizado na Figura 4.

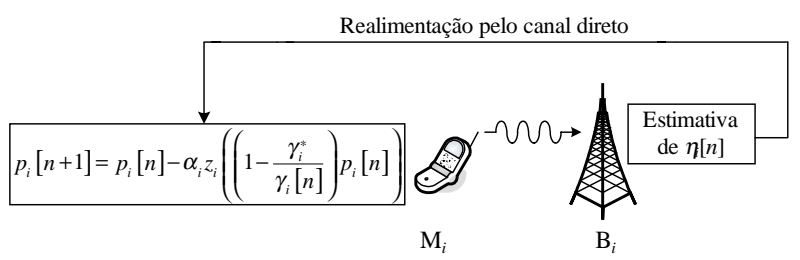

Figura 4. Princípio de funcionamento de um DPCA.

É possível mensurar $\gamma_{i}[n]$ sem o conhecimento efetivo das informações relativas aos usuários interferentes, as quais influenciam em seu valor de acordo com a eq. (2). Assim, (18) depende apenas de parâmetros locais, permitindo que o controle de potência como um todo funcione de forma distribuída, ou seja, que cada um dos $K$ elos $\mathrm{M}_{i} \mathrm{~B}_{i}$ realize isoladamente o respectivo controle de potência (ULUKUS; YATES, 1998). Justifica-se, portanto, a denominação algoritmo de controle de potência distribuído (DPCA) para o conjunto de recursões em (18). 
Em contraste à estratégia distribuída, o método centralizado para resolver o problema de alocação ótima consiste em reunir todos os elementos da matriz $\mathbf{H}$ e do vetor $\boldsymbol{\eta}$ em alguma entidade que execute a operação em (4) e posteriormente "entregue" os valores ótimos das potências. Com isso, o esquema centralizado necessita da estimação de cada elemento de $\mathbf{H}$ e de $\boldsymbol{\eta}$, do tráfego dos valores destes elementos através do sistema de comunicação e, finalmente, de uma inversão matricial. Portanto, a complexidade de implementação da metodologia centralizada é acentuadamente maior do que a distribuída. Quando o número de usuários ativos no sistema multicelular for elevado, a quantidade de operações necessárias para a inversão matricial, o volume de estimações e o tráfego na rede sem fio desses valores estimados atingem proporções que tornam impraticável a estratégia centralizada.

\section{A. DPCAs Comparados}

O DPCA desenvolvido por Foschini e Miljanic (FOSCHINI; MILJANIC, 1993), aqui denominado DPCA clássico, é obtido por meio de uma substituição simples:

$$
z_{i}(x)=x, \quad \alpha_{i}=\alpha, i=1, \ldots, K,
$$

em (18); portanto, o processo iterativo para o DPCA clássico resulta:

$$
p_{i}[n+1]=p_{i}[n]-\alpha\left(1-\frac{\gamma_{i}^{*}}{\gamma_{i}[n]}\right) p_{i}[n], \quad i=1, \ldots, K
$$

No trabalho publicado por (UYKAN; KOIVO, 2004) sugere-se que o controle de potência equivale a um problema de projeto de controlador, conforme o modelo contínuo da Figura 3. Para sua síntese, simplesmente substitui-se:

$$
z_{i}(x)=\tanh \left(\frac{x}{2}\right), \quad \alpha_{i}=\alpha, i=1, \ldots, K,
$$

em (18). Assim, o DPCA sigmoidal (UYKAN; KOIVO, 2004) é dado por:

$$
\begin{gathered}
p_{i}[n+1]=p_{i}[n]-\alpha \tanh \left(\frac{1}{2}\left(1-\frac{\gamma_{i}^{*}}{\gamma_{i}[n]}\right) p_{i}[n]\right), \\
i=1, \ldots, K
\end{gathered}
$$

Observe que o desenvolvimento feito em (UYKAN; KOIVO, 2004) inclui ainda uma constante multiplicativa ajustável no argumento da função $\tanh ($.$) .$

A utilização de função sigmoidal resulta em convergência quadrática na vizinhança do ponto ótimo (UYKAN; KOIVO, 2004) e desde que $\rho_{\mathbf{H}}<1$, o algoritmo sigmoidal converge para qualquer vetor de potência inicial.

\section{DPCA Proposto}

O modelo dinâmico atribuído a P.F.Verhulst, estabelecido em (VERHULST, 1838) e comentado em (MONTEIRO, 2002), é dado por:

$$
\dot{p}=z(p)=p\left(1-\frac{p}{p^{*}}\right)
$$

Verhulst formulou esta equação na tentativa de descrever a evolução temporal do número de indivíduos, denotado por $p(t)$, de uma determinada espécie biológica. Seu modelo asssume que o alimento disponível e o espaço físico ocupado são finitos. Essa limitação de recursos impede que a população cresça de maneira ilimitada. Tais considerações são levadas em conta no termo que provoca diminuição da taxa de crescimento conforme a população aumenta. Integrando-se (23) analiticamente, obtém-se:

$$
p(t)=\frac{\exp (t) p(0) p^{*}}{p^{*}+p(0)[\exp (t)-1]}
$$

O comportamento assintótico desta solução é dado por: 


$$
\lim _{t \rightarrow+\infty} p(t)=p^{*}
$$

para qualquer condição inicial $p(0)$ estritamente positiva, conforme evidenciado na Figura 5.

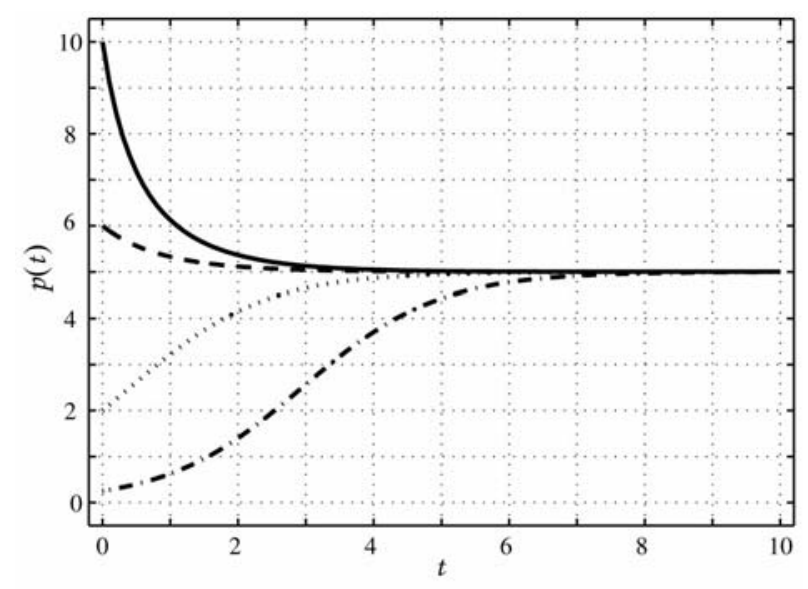

Figura 5. Curvas $p(t)$ parametrizadas por diferentes condições iniciais.

Quando $p(0)$ for maior que $p^{*}$, o sinal de $d p / d t$ será negativo, e $p(t)$ decrescerá de forma que $p(+\infty)=p^{*}$. Caso contrário, $d p / d t$ será positivo, e $p(t)$ aumentará monotonicamente em direção ao seu valor assintótico (VERHULST, 1838) .

Supondo que existam várias espécies sob análise, é conveniente que se reescreva (17) em um formato matricial:

$$
\dot{p}=z(p)=p \otimes\left(u-p \Theta p^{*}\right)
$$

$\operatorname{com} \mathbf{p}=\left[\begin{array}{ll}p_{1} \mathrm{~L} & p_{K}\end{array}\right]^{T}, \mathbf{u}=\left[\begin{array}{ll}1 \mathrm{~L} & 1\end{array}\right]^{T}$ e $\mathbf{p}^{*}=\left[\begin{array}{ll}p_{1}^{*} \mathrm{~L} & p_{K}^{*}\end{array}\right]^{T}$. Os operadores empregados em (26) representam as operações elementares $[\mathbf{A} \otimes \mathbf{B}]_{i j}=[\mathbf{A}]_{i j}[\mathbf{B}]_{i j} \quad \mathrm{e}$ $[\mathbf{A} \Theta \mathbf{B}]_{i j}=[\mathbf{A}]_{i j} /[\mathbf{B}]_{i j}$, sendo $\mathbf{A}$ e $\mathbf{B}$ matrizes com dimensões iguais.

Impondo que a evolução do vetor de potências possa ser regida pelo modelo biológico de (26) e admitindo-se que $\mathbf{p}$ esteja suficientemente próximo de $p^{*}$ pode-se escrever, a partir de (3):

$$
\mathbf{p}^{*} \approx \mathbf{H p}+\boldsymbol{\eta}
$$

Substituindo (27) no modelo de Verhulst, adaptado à forma matricial em (26), obtém-se:

$$
\dot{\mathbf{p}}=\mathbf{p} \otimes[\mathbf{u}-\mathbf{p} \Theta(\mathbf{H p}+\boldsymbol{\eta})]
$$

Portanto a função z(.) resulta:

$$
\mathbf{z}(-(\mathbf{I}-\mathbf{H}) \mathbf{p}+\boldsymbol{\eta})=\mathbf{p} \otimes[\mathbf{u}-\mathbf{p} \Theta(\mathbf{H p}+\boldsymbol{\eta})]
$$

Neste caso, o formato do mapeamento $\mathbf{y}=\mathbf{z}(\mathbf{x})$ depende da matriz $\mathbf{H}$ e do vetor $\boldsymbol{\eta}$. Considerando o ponto $K$-dimensional $\mathbf{x}_{0}=-\left(\mathbf{I}-\mathbf{H}_{0}\right) \mathbf{p}_{0}+\boldsymbol{\eta}_{0}$, obtém-se, após a aplicação da função $\mathbf{z}($.$) , o ponto$ $\mathbf{y}_{0}=\mathbf{p}_{0} \otimes\left[\mathbf{u}-\mathbf{p}_{0} \Theta\left(\mathbf{H}_{0} \mathbf{p}_{0}+\boldsymbol{\eta}_{0}\right)\right]$. Alterando $\mathbf{H}_{0}$ para $\mathbf{H}_{1}$ e $\eta_{0}$ para $\boldsymbol{\eta}_{1}$, é sempre possível, evidentemente, encontrar um vetor $\mathbf{p}_{1}$ de forma que $\mathbf{x}_{0}=-\left(\mathbf{I}-\mathbf{H}_{0}\right) \mathbf{p}_{0}+\boldsymbol{\eta}_{0}=-\left(\mathbf{I}-\mathbf{H}_{1}\right) \mathbf{p}_{1}+\boldsymbol{\eta}_{1}=\mathbf{x}_{1} . \quad$ Sendo $\mathbf{x}_{1}$ assim definido, tem-se $\mathbf{y}_{1}=\mathbf{z}\left(\mathbf{x}_{1}\right)=\mathbf{p}_{1} \otimes\left[\mathbf{u}-\mathbf{p}_{1} \Theta\left(\mathbf{H}_{1} \mathbf{p}_{1}+\boldsymbol{\eta}_{1}\right)\right] \neq \mathbf{y}_{0}$. Portanto, para o DPCA proposto, a modificação da matriz $\mathbf{H}$ e/ou do vetor $\boldsymbol{\eta}$ altera a função $\mathbf{y}=\mathbf{z}(\mathbf{x})$. No DPCA clássico e no sigmoidal, o mapeamento $\mathbf{y}=\mathbf{z}(\mathbf{x})$ é imutável.

Analogamente aos DPCA's clássico e sigmoidal, o comportamento do DPCA proposto pode ser sintetizado a partir de um sistema de controle. Entretanto, para este novo DPCA, o diagrama de controle apresentado na Figura3 é insuficiente e deve ser modificado de forma a incorporar a variabilidade do mapeamento $\mathbf{y}=\mathbf{z}(\mathbf{x})$. Esta modificação está representada na Figura 6 pela presença do bloco e seta tracejados, os quais estão indicando que a função $z_{i}$ (.) sofre influência de cada $h_{i j, i \neq j}$ e de $\eta_{i}$.

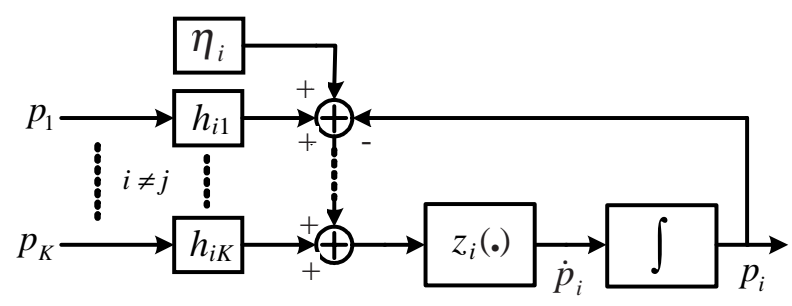

Figura 6. Comportamento sistêmico do DPCA proposto 
Discretizando (28) pelo método NIE, obtém-se:

$\mathbf{p}(n+1)=\mathbf{F}(\mathbf{p}(n))=\mathbf{p}(n)+\alpha \mathbf{p}(n) \otimes\{\mathbf{u}-\mathbf{p}(n) \Theta[\mathbf{H p}(n)+\boldsymbol{\eta}]\}$

Portanto, calcula-se o elemento $i$ do vetor $\boldsymbol{F}(p)$ por:

$$
F_{i}(\mathbf{p})=(1+\alpha) p_{i}-\frac{\alpha}{\gamma_{i}^{*}} \mu_{i}(\mathbf{p}) p_{i}^{2}
$$

onde $\quad \mu_{i}(\mathbf{p})=\frac{g_{i i}}{\sum_{j \neq i} g_{i j} p_{j}+\sigma_{i}^{2}}$

A equação matricial (30) é equivalente ao conjunto de equações escalares:

$$
p_{i}(n+1)=(1+\alpha) p_{i}(n)-\alpha\left[\frac{\gamma_{i}(n)}{\gamma_{i}^{*}}\right] p_{i}(n), i=1, \ldots, K
$$

As equações em (32) consistem na versão implementável do DPCA proposto. Salienta-se que o diagrama de controle da Figura 6 é apenas uma forma sistemática de visualizar como ocorre a evolução da potência transmitida, e não corresponde à estrutura que deve ser efetivamente implementada na prática. Observa-se que a implementação direta do esquema da Figura 6 exigiria o conhecimento exato de cada $h_{i j, i \neq j}, \eta_{i}$ e $z_{i}($.) e, conseqüentemente, seria um procedimento centralizado. De fato, se $h_{i j, i \neq j}, \eta_{i}$ e $z_{i}($.$) fossem conhecidos exatamente e se a$ estimação da $\operatorname{SINR} \gamma_{i}(n)$ fosse perfeita em cada iteração $n$ de (32), o sistema de controle da Figura 6 produziria o mesmo resultado que (32).

Observa-se, em (32), que a atualização da potência transmitida de cada usuário depende apenas de parâmetros referentes ao mesmo, ou seja, da sua própria SINR estimada, do seu nível de potência transmitido na iteração anterior e de sua meta de QoS. Esse aparente desacoplamento entre os usuários decorre, evidentemente, do fato de se estimar a SINR diretamente e não efetuar seu cálculo pela relação que a define, equação (2). A obtenção da SINR aplicando-se (2) exigiria processos dispendiosos de estimação para os ganhos de canal dos interferentes e para o ganho do usuário de interesse, tornando a implementação do algoritmo mais complexa.

As considerações mencionadas no parágrafo anterior são típicas de algoritmos distribuídos. Entretanto, o DPCA proposto, definido pelas iterações em (32), apresenta uma característica singular: no termo entre colchetes, a SINR estimada está no numerador da divisão. Esta propriedade torna a sensibilidade do algoritmo face a erros de estimação independente do nível verdadeiro da SINR. Sabe-se que função do tipo $\varphi=\kappa \gamma^{-1}$ (SINR no denominador é inerente aos algoritmos distribuídos propostos na literatura atual, como o clássico (FOSCHINI; MILJANIC, 1993) e o sigmoidal (UYKAN; KOIVO, 2004) possui incremento diferencial $\mathrm{d} \varphi=$ $\kappa \gamma^{-2} \mathrm{~d} \gamma$, ou seja, o montante da variação de $\varphi$ para pequenos desvios de $\gamma$ depende do valor real de $\gamma$; enquanto que para a função do tipo $\varphi=\kappa \gamma$ (SINR no numerador, caso do DPCA proposto), tem-se $\mathrm{d} \varphi=$ $\kappa \mathrm{d} \gamma$, não havendo influência, portanto, do nível verdadeiro de $\gamma$ sobre a variação de $\varphi$. No algoritmo sigmoidal (UYKAN; KOIVO, 2004), observa-se que o resultado da operação na qual $\gamma$ está no denominador é ainda argumento de uma função do tipo tangente hiperbólica. Evidentemente, esta função hiperbólica influencia, mas não anula, a relação entre o valor real de $\gamma$ e o comportamento do algoritmo na presença de medidas imperfeitas de SINR.

\section{Resultados Numéricos}

Esta seção traz uma análise numérica comparativa dos algoritmos clássico (FOSCHINI; MILJANIC, 1993) e sigmoidal (UYKAN; KOIVO, 2004) com o proposto em termos das principais características de um algoritmo de controle de potência distribuído:

a) o quão próxima da solução do problema de alocação está a solução encontrada pelo algoritmo;

b) consumo médio de potência por usuário quando o 
algoritmo atinge o regime;

c) número de atualizações para o algoritmo atingir a condição de regime;

d) complexidade de implementação.

A característica a) pode ser quantificada por meio do erro quadrático médio normalizado (NSE) na condição de regime. A definição do NSE é dada por (ULUKUS; YATES, 1998):

$$
\operatorname{NSE}(n)=E\left[\frac{\left\|\mathbf{p}(n)-\mathbf{p}^{*}\right\|^{2}}{\left\|\mathbf{p}^{*}\right\|^{2}}\right]
$$

onde $\|\cdot\|^{2}$ denota o quadrado da distância euclidiana em relação à origem e $E[$.$] o operador esperança.$ O consumo médio de potência por usuário na $n$-ésima iteração é calculado por:

$$
\operatorname{PM}(n)=E\left[\frac{1}{K} \sum_{i=1}^{K} p_{i}(n)\right]
$$

O valor de (34) pode ser verificado quando o algoritmo atinge o regime quantifica a característica b). O número de iterações necessárias para o $\operatorname{NSE}(n)$ ficar confinado em um intervalo estreito préestabelecido quantifica a característica c). Finalmente, uma forma simples de quantificar a característica d) consiste em obter o total de somas, subtrações, divisões, multiplicações e acessos a valores em tabelas necessários à realização de uma atualização do algoritmo multiplicado pelo número de atualizações necessárias para a convergência, $m$.

\section{B. Parâmetros de Simulação}

As simulações foram realizadas em ambiente Matlab 6.5 para $K=5,10$ e 15 usuários em um sistema
DS/CDMA. Admite-se que a seguinte condição seja sempre satisfeita.

$$
m L T_{b}<<(\Delta t)_{c}
$$

onde $T_{b}$ e $(\Delta t)_{c}$ são, respectivamente, o período de bit de informação e o tempo de coerência do canal ${ }^{4}$ (SKLAR, 1997). Essa condição faz parte do processo de estimação da SINR, implicando que cada atualização de potência realizada pelo DPCA ocorre em intervalos de pelo menos $L$ bits. As recursões em (21), (26) ou em (32) devem convergir para a solução ótima do problema de alocação, antes que cada ganho de canal $g_{i i}$ experimente significativa alteração ${ }^{5}$.

Note-se que satisfeita (35), as matrizes de ganhos resultam estáticas no intervalo de tempo referente a um único processo de convergência. Nas simulações realizadas, os elementos de cada matriz $\mathbf{G}$ possuem valores intermediários entre os usados em (UYKAN; KOIVO, 2004), eq. (26) e (CHAI et al, 2004), (Ex. 1). As metas de QoS utilizadas estão entre $4 \mathrm{~dB}$ e 6 dB, faixa baseada em (UYKAN; KOIVO, 2004) e (ULUKUS; YATES, 1998). A potência de ruído no receptor é $\sigma_{i}^{2}=0,1 p W, i=1, \cdots, K$ a todos os usuários, valor também usado em (UYKAN; KOIVO, 2004), exemplo 1.

$\mathrm{O}$ algoritmo proposto e o de Foschini et al foram simulados para $\alpha$ 's (passos de integração numérica) positivos não maiores do que a unidade, pois ambos divergem para outros valores de passo (FOSCHINI; MILJANIC, 1993). De acordo com (UYKAN; KOIVO, 2004), o sigmoidal converge para qualquer $\alpha$ positivo. Entretanto, foram adotados $\alpha$ 's não maiores do que 1 para o mesmo devido à excessiva degradação do desempenho em termos de proximidade da solução ótima em regime à medida que $\alpha$ aumenta além da unidade.

\footnotetext{
4 Corresponde ao intervalo de tempo na qual as características do canal não experimentam variação expressiva.

5 A seleção de um $L$ apropriado é fundamental. Se for escolhido um valor pequeno, as atualizações de potência serão mais freqüentes e a convergência será mais rápida. Entretanto, realimentações freqüentes no canal direto diminuem a capacidade do sistema, pois "consomem" banda.
} 
$\mathrm{Na}$ prática, as estimativas das SINR's nas estações rádio base não são perfeitas, no sentido de que os valores obtidos no processo de estimação continuam a apresentar caráter aleatório. Para incorporar esta situação realista às simulações, adicionou-se um erro aleatório às SINR's calculadas a cada iteração. Considerou-se que a razão entre o valor estimado da SINR e o verdadeiro vale $(1+\varepsilon)$, onde $\varepsilon$ é uma variável aleatória uniformemente distribuída no $[-\delta ;+\delta]$. Realizaram-se as simulações para os seguintes $\delta$ 's: 0,$01 ; 0,05 ; 0,1 ; 0,2$; e 0,25 . Esses valores também foram utilizados em (UYKAN; KOIVO, 2004), exemplo 1.

Em todas as simulações, os três algoritmos iterativos foram inicializados com o vetor de potências $\mathbf{p}(0)=[0,1 ; 0,1 ; \ldots ; 0,1]^{\mathrm{T}} \mathrm{pW}$.

\section{Resultados de Simulação}

O comportamento da distância euclidiana em relação ao vetor ótimo versus iteração para os três algoritmos, considerando o caso de $K=2$ usuários, é mostrado nas Figura 7, 8 e 9. Parâmetros de interesse podem ser extraídos da inspeção dessa curva: dinâmica de convergência (oscilante, monotônica etc), número de iterações necessárias para se atingir a condição de regime e a própria distância euclidiana em regime.

As condições de desvanecimento foram consideradas estáticas e definidas pela matriz de ganhos de canal:

$$
\mathbf{G}=\left[\begin{array}{ll}
0,5782 & 0,1000 \\
0,2833 & 0,8870
\end{array}\right],
$$

resultado das atenuações promovidas pelo canal e as correlações cruzadas entre as seqüências de espalhamento Adicionalmente, para cada dos dois usuários, admitu-se um índice de QoS estabelecido por $10 \log _{10}\left(\gamma_{i}^{t g t}\right)=6 \mathrm{~dB}$. Este cenário (meta de QoS, potência do AWGN e matriz G) é idêntico ao utilizado em (UYKAN; KOIVO, 2004), resultando no raio espectral $\rho_{\mathbf{H}} \approx 0,94$.
Observa-se que, caso fossem utilizados níveis de QoS inferiores a $4,95 \mathrm{~dB}$, com as demais condições inalteradas, obter-se-ia $R_{G_{r}}^{\max } \mathrm{G}_{c}<1$. Dessa forma, o teste baseado nos GC seria conclusivo sobre a viabilidade do problema de alocação, e não necessitaria, portanto, do cálculo efetivo do autovalor de Perron-Frobenius. Por outro lado, se os níveis de QoS exigidos fossem superiores a 6,29dB, mantendose as demais características do cenário, ter-se-ia um problema não factível, uma solução irrealizável, $\rho_{\mathbf{H}}>1$.

Para facilitar o estudo das propriedades de convergência, é conveniente obter uma curva média $\bar{d}_{\alpha}(n)$ a partir de várias funções amostra $d_{\alpha}(n)$. Considerando $\delta=0,2$, ou seja, em cada iteração escolhe-se de forma não tendenciosa um erro de estimativa que não exceda $20 \%$ do valor real da SINR atualizada, simulou-se os três DPCA's descritos. Cada curva $\bar{d}_{\alpha}(n)$ foi obtida tomando-se a média em 5000 funções amostra $d_{\alpha}(n)$. Este procedimento foi repetido para diferentes valores do parâmetro $\alpha$. Nas simulações do DPCA clássico, os valores de $\alpha$ foram escolhidos no intervalo [0;1], pois foi mostrado analiticamente em (FOSCHINI; MILJANIC, 1993) que nesta faixa de valores ocorre convergência. Em (UYKAN; KOIVO, 2004) também foi mostrado de forma analítica, que o DPCA sigmoidal converge para qualquer valor $\alpha>0$. Nesse caso irrestrito, os limites para os valores de $\alpha$ foram definidos de modo que a velocidade de convergência e/ou a $\bar{d}_{\alpha}(n)$ não fossem excessivamente piores que os resultados análogos dos DPCA's clássico e proposto. A distância euclidiana em relação ao vetor ótimo atingida pelo DPCA proposto, Figura 7, mostrou-se estritamente menor que a obtida pelo DPCA clássico, Figura 8. Diferentemente do algoritmo clássico, o proposto converge de forma monotônica. A velocidade máxima de convergência do DPCA clássico ocorre quando $\alpha=1$ e a do proposto, nas condições simuladas, quando $\alpha=0,65$. Mesmo nessas condições de sistema, a velocidade máxima do DPCA clássico, em torno de 100 iterações, é aproximadamente a mesma que a do proposto. 


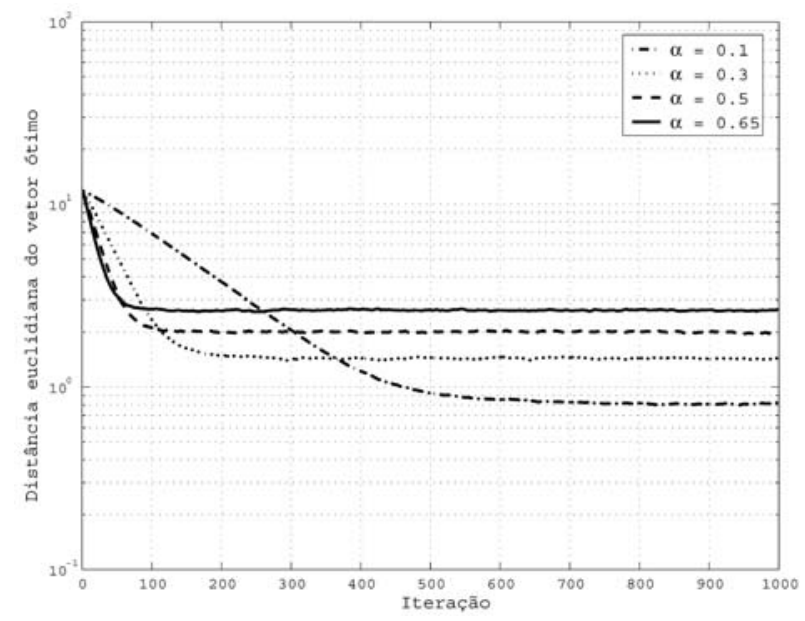

Figura 7. Distância euclidiana em cada iteração do DPCA proposto.

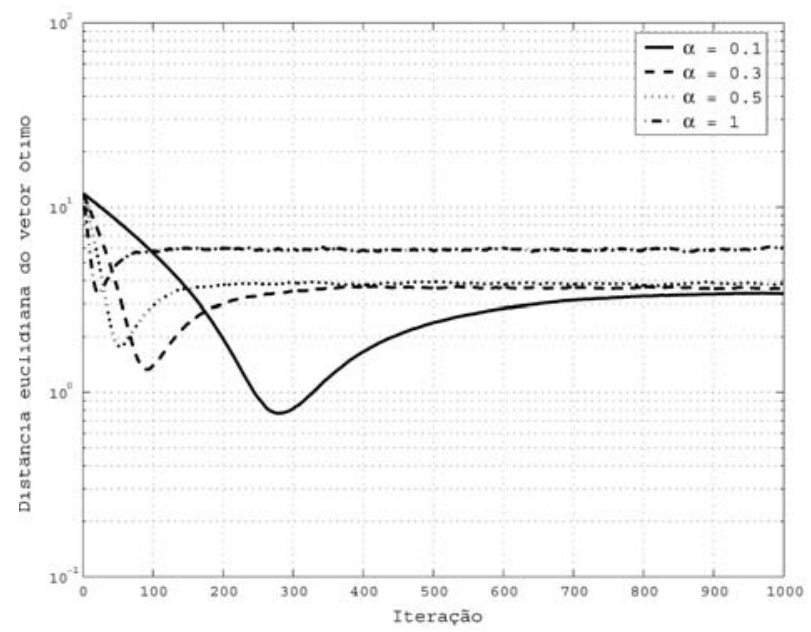

Figura 8. Distância euclidiana em cada iteração do DPCA clássico.

O comportamento da distância euclidiana do algoritmo sigmoidal em relação ao vetor ótimo versus iteração é mostrado na Figura 9. Adicionalmente, verificou-se que o processo de convergência do DPCA sigmoidal torna-se monotônico à partir de $\alpha \approx 4$ (não mostrado aqui). Na situação em que o DPCA proposto e o sigmoidal possuem velocidades de convergência próximas, o DPCA proposto atinge distância euclidiana assintótica menor: verifique na Figura 7 o valor em regime atingido pelo DPCA proposto quando $\alpha=0,65$ e na Figura 9 aquele obtido pelo sigmoidal $\operatorname{com} \alpha=2,5$.

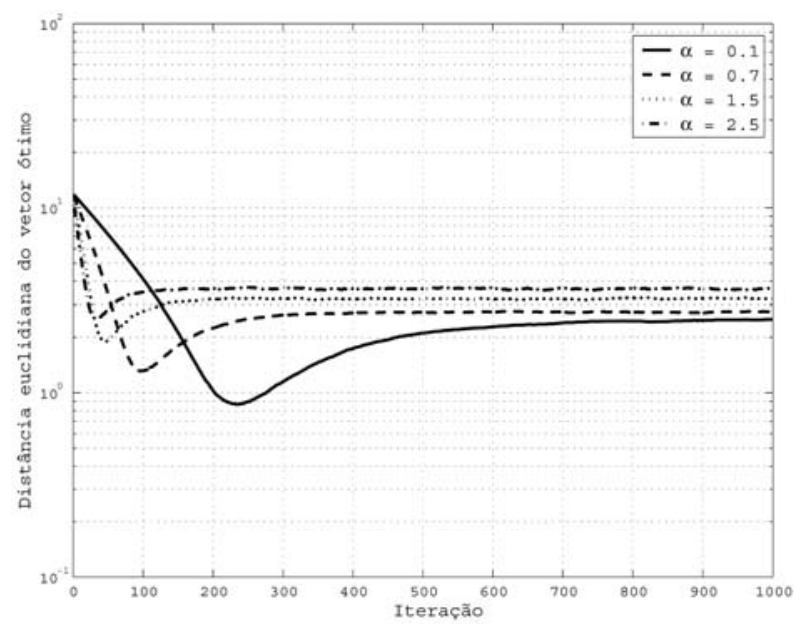

Figura 9. Distância euclidiana em cada iteração do DPCA sigmoidal.

Em seguida, o comportamento dos três algoritmos foi analisado para carregamentos maiores, $K=5,10$ e 15 usuários, obtendo-se a curva $\operatorname{NSE}(n)$, eq. (33), a partir da geração de 1000 funções amostra $\mathbf{p}(n)$ para cada algoritmo, cada qual executando 2000 iterações. Sintetizou-se a curva $\operatorname{PM}(n)$, eq. (34), de cada algoritmo de forma análoga. Obteve-se, então, uma média com os últimos 500 pontos de cada curva $\operatorname{NSE}(n)$ e $\operatorname{PM}(n)$. Estes valores representam, respectivamente, o NSE e o PM em regime. Finalmente, com esses valores em regime calcularam-se as seguintes razões:

$$
\begin{aligned}
& \operatorname{NSER}_{\text {Foschini }}=\frac{\mathrm{NSE}_{\text {Proposto }}^{\text {regime }}}{\mathrm{NSE}_{\text {Foschini }}^{\text {regie }}} \\
& \mathrm{NSER}_{\text {Sigmoidal }}=\frac{\mathrm{NSE}_{\text {Proposto }}^{\text {regime }}}{\mathrm{NSE}_{\text {Sigmoidal }}^{\text {regime }}} \\
& \operatorname{PMR}_{\text {Foschini }}=\frac{\mathrm{PM}_{\text {Proposto }}^{\text {regime }}}{\mathrm{PM}_{\text {Foschini }}^{\text {regie }}} \\
& \mathrm{PMR}_{\text {Sigmoidal }}=\frac{\mathrm{PM}_{\text {Proposto }}^{\text {regime }}}{\mathrm{PM}_{\text {Sigmoidal }}^{\text {regime }}}
\end{aligned}
$$

Repetiu-se o procedimento descrito acima para conjuntos $(\alpha, \delta, K)$ representativos. Os resultados estão mostrados nas Figura 11 a 16. Para cada $(\alpha$, 
$\delta, K)$, existem três curvas $\operatorname{NSE}(n)$, cada qual referente a um algoritmo. Considere o máximo desvio, nos últimos 500 pontos, de cada uma dessas três curvas em relação aos respectivos valores de regime $^{6}$. Dentre esses três valores máximos, selecionouse o maior que, em seguida, foi multiplicado por 2 . $\mathrm{O}$ valor resultante deste procedimento define o máximo desvio que cada uma das três curvas $\operatorname{NSE}(n)$ pode ter em regime. Assim, o número de iterações necessárias para a convergência de cada algoritmo foi definido como aquele a partir do qual o desvio da correspondente curva $\operatorname{NSE}(n)$ em relação ao seu valor de regime é sempre menor que esse máximo desvio. Note-se que cada tripla $(\alpha, \delta, K)$ resulta em um desvio máximo diferente.

Assim, o número de iterações necessário para a convergência de cada algoritmo depende de $(\alpha, \delta$, $K)$. Entretanto, o parâmetro determinante da velocidade de cada algoritmo é $\alpha$, pois constitui o próprio passo de integração numérica. Para cada $\alpha$, realizou-se a média em $\delta$ e em seguida em $K$. Os resultados obtidos são mostrados nas curvas da Figura 10. Denota-se $m_{\text {avg }}$ o número médio de iterações para a convergência de cada algoritmo.
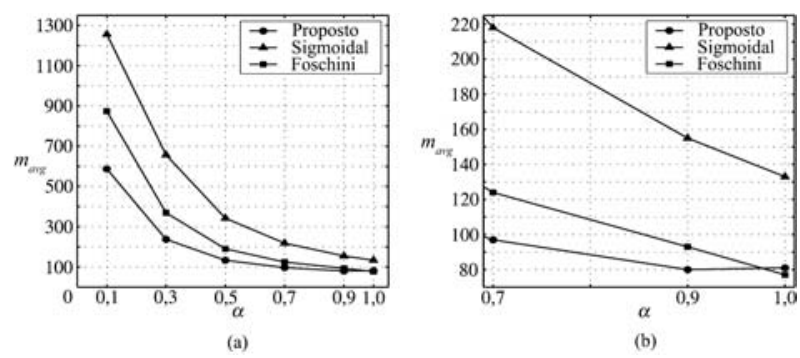

Figura 10. Número médio de iterações para a convergência em função de $\alpha$.

Verifica-se na Figura 13 que o algoritmo proposto possui desempenho superior em termos de número médio de iterações em relação aos outros dois, exceto para $\alpha=1$. Nessa situação, o de Foschini et al. mostra-se superior aos demais, porém isso ocorre de forma marginal em relação ao proposto. Observese que a discrepância de ambos em relação ao proposto é maior conforme $\alpha$ decresce. Se existir um limite superior para $m_{\text {avg }}$, eq. (35), é possível utilizar, com vantagem, um passo $\alpha$ menor para o proposto. Isso é importante, pois sabe-se que menores passos de integração numérica resultam em maior proximidade, em regime, da solução ótima.

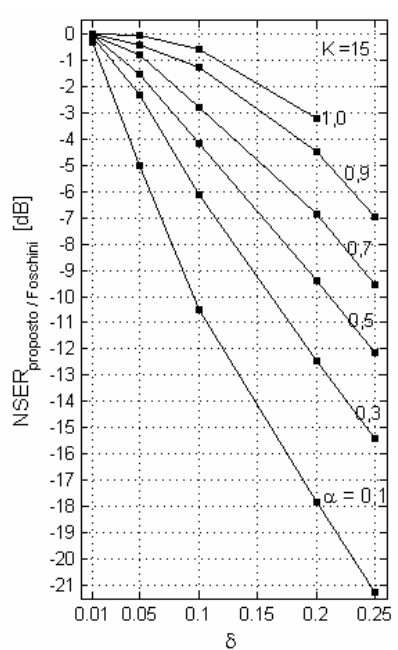

(a)

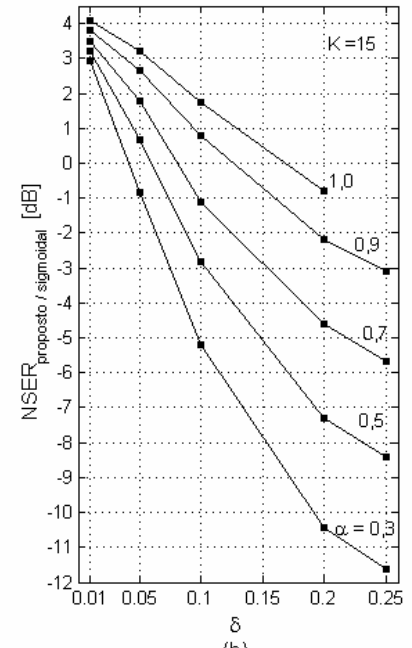

(b)
Figura 11. Razão em termos de NSE em regime para $K=15$ : a) proposto / Foschini; b) proposto / sigmoidal.
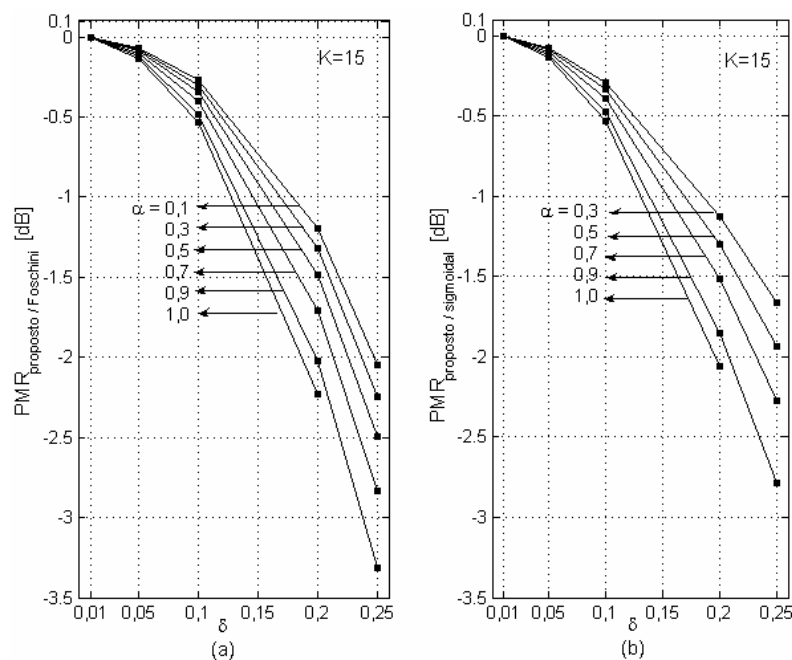

(b)

Figura12. Razão em termos de PM em regime para $K=15$ : a) proposto / Foschini; b) proposto / sigmoidal.

${ }^{6}$ Ressalte-se que a média nos últimos 500 pontos foi adotada como representante do valor de regime. 

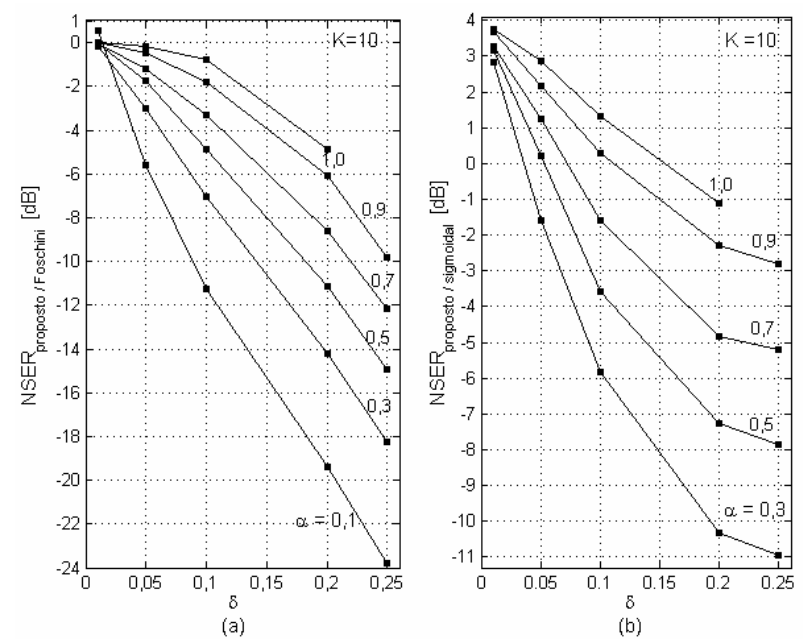

Figura 13. Razão em termos de NSE em regime para $K=10$ : proposto / Foschini; b) proposto / sigmoidal

Conforme as Figura 12, 14 e 16, o DPCA proposto, para qualquer $\alpha$ e $\delta$, resulta em menor consumo de potência por usuário (PM em regime) em relação aos outros dois. Observa-se que essa vantagem em termos de PM em regime tende a ser maior à medida que o erro de estimação e o passo $\alpha$ aumentam. Essa economia de potência por parte do algoritmo proposto constitui efetivamente uma vantagem se o vetor de potências em regime fornecido pelo mesmo não ficar muito distante (alto NSE em regime) do vetor ótimo. Já os resultados nas Figura 11, 13 e 15 comprovam que na maioria das situações o algoritmo proposto atinge um menor NSE em regime; nas poucas situações restantes, o NSE em regime é comparável aos valores de NSE em regime dos outros dois DPCA's.

Verifica-se que o NSER Sigmoidal $(\mathrm{dB})$ apresenta uma tendência positiva ao se diminuir $\delta$, tendendo a superar o algoritmo proposto em termos de NSE em regime apenas para baixos erros de estimação. Observe-se que o quão baixo deve ser o $\delta$ para que NSER $_{\text {Sigmoidal }}(\mathrm{dB})$ torne-se positivo depende

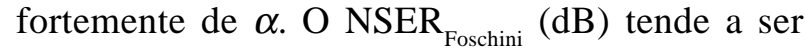
estritamente não positivo quando o sistema está carregado com mais de 2 usuários. Para $K>2$, a vantagem do proposto em relação ao de Foschini $e t$ al em termos de NSE em regime aumenta estritamente com $\delta$. Verifica-se, ademais, que a diminuição do passo $\alpha$ tende a beneficiar mais o DPCA proposto que os outros dois, em termos de NSE em regime.
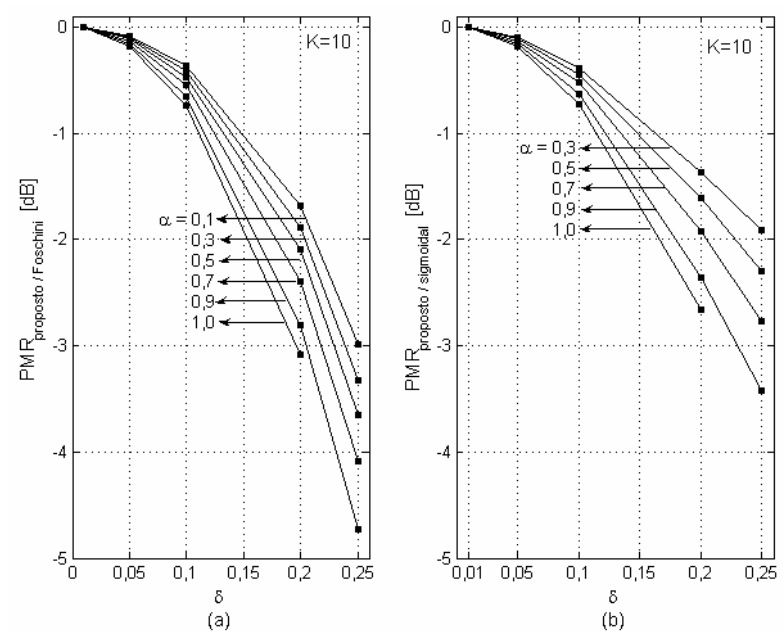

Figura 14. Razão em termos de PM em regime para $K=10$ : (a) proposto / Foschini; b) proposto / sigmoidal.
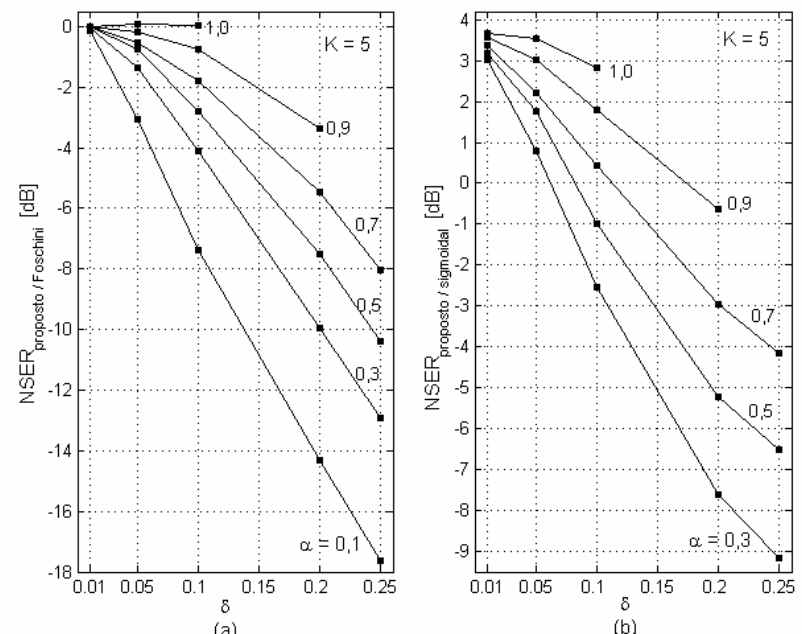

Figura 15. Razão em termos de NSE em regime para $K=5$ : a)proposto / Foschini; b) proposto / sigmoidal

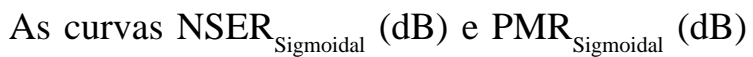
quando $\alpha=0,1$ não foram incluídas nas Figura 11 a 16 , pois o algoritmo sigmoidal não atingiu a situação de regime após 2000 iterações. A diminuição da quantidade de pontos nessas Figs ocorre à medida que o número de usuários decresce (baixos carregamentos) e isso se deve à tendência do algoritmo proposto não convergir quando há, 
simultaneamente, baixo carregamento, elevado erro de estimação e passo $\alpha$ próximo da unidade.
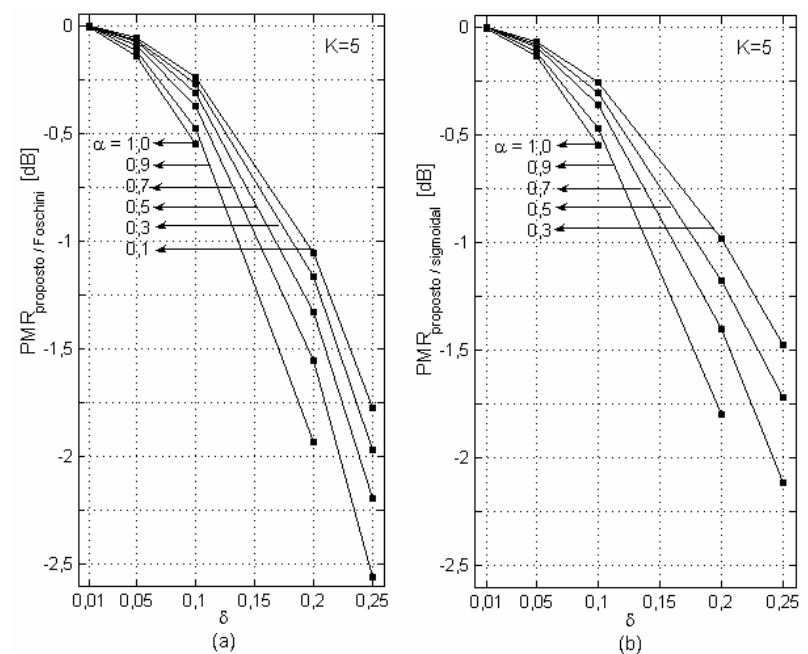

Figura 16. Razão em termos de PM em regime para $K=5$ : (a) proposto / Foschini; b) proposto / sigmoidal

No que tange à complexidade, como no método distribuído cada elo usuário-estação rádio base, $M_{i} B_{i}$, realiza isoladamente suas atualizações, ou seja, o controle de potência como um todo é realizado por $K$ processadores em paralelo e cada qual executa apenas operações escalares. A análise, então, reduzse ao estudo de uma iteração. Conclui-se que uma iteração no DPCA proposto e no de Foschini et al. possuem a mesma complexidade. Já uma iteração no sigmoidal exige, adicionalmente, acesso a uma tabela para o cômputo da função $\tanh ($.$) ; portanto, o$ DPCA sigmoidal possui maior complexidade de implementação.

\section{Conclusões}

Apresentou-se e analisou-se um novo algoritmo DPCA para sistemas CDMA em termos das métricas adotadas na comparação de desempenho. O DPCA proposto mostrou-se superior a dois algoritmos bastante conhecidos na literatura em situações práticas de interesse, isto é, quando ocorrer erros nas estimativas de $\gamma_{i}$ associado a sistemas mediana e altamente carregados.
O Teorema dos GC aliado à estrutura matricial particular do problema de alocação resultou na definição de um conjunto de critérios suficientes para a viabilidade. Mostrou-se que todo sistema com norma-s, $\mathrm{s}=1$, de cada vetor-linha de $\mathbf{H}$ ou $\mathbf{H}^{\mathrm{T}}$ menor do que a unidade é factível. Verificar essas condições exige pouco esforço computacional em relação ao necessário para a estimação efetiva do autovalor de Perron-Frobenius. Adicionalmente, a metodologia proposta permite avaliar de uma forma simples a tendência sobre a viabilidade de solução para o problema de alocação de potência conforme sejam modificados os parâmetros que definem $h_{i j}$, ou seja, a atenuação provocada pelo canal sobre o sinal transmitido $j$, a correlação cruzada entre as seqüências de espalhamento $i$ e $j$, e o nível de QoS designado ao usuário $j$.

O diagrama de controle da Figura 3 é geral e constitui uma forma sistemática de descrever o comportamento dinâmico dos diversos DPCA's descritos na literatura. Observe que os três algoritmos analisados neste artigo são estruturas particulares do sistema da Figura 3. Essa forma de descrição genérica pode facilitar eventuais comparações e o processo de síntese de novos algoritmos distribuídos.

Os algoritmos distribuídos simulados na ausência de erro de estimação apresentaram resultados que na prática podem ser considerados idênticos. Entretanto, quando as medidas das SINR's não foram consideradas perfeitas, correspondendo aos casos práticos de interesse em engenharia, as diferenças entre os DPCA's mostraram-se substanciais. O DPCA proposto alcançou a condição de regime mais rapidamente e de forma assintótica. Adicionalmente, o DPCA proposto atingiu menor distância euclidiana do ponto $K$-dimensional ótimo quando as velocidades de convergência dos três algoritmos mostraram-se próximas. Em suma, o DPCA proposto resultou em pronunciada robustez aos erros de estimação aleatórios quando comparado aos algoritmos clássico e sigmoidal. 


\section{Referências}

CHAI, C. C; LU, Y.; CHEW, Y. H.; TJHUNG, T. T. A Unified Framework for Transmitter Power Control in Cellular Radio Systems. ETRI Journal, Daejeon, v.26, n.5, p.423-431, oct. 2004.

FOSCHINI, G.; MILJANIC, Z., A Simple Distributed Autonomous Power Control Algorithm and its Convergence, IEEE Transactions on Vehicular Technology, New York, v.42, n.4, p. 641-646, nov. 1993.

MEYER, C. D. Matrix Analysis and Applied Linear Algebra. Philadelphia: SIAM Publications, 2000.

MONTEIRO, L. H. A. Sistemas Dinâmicos. São Paulo: Ed.da Física, 2002.

PRESS, W. H.; TEUKOLSKY, S. A.; VETTERLING, W.T.; FLANNERY, B. P. Numerical Recipes in C: The Art of Scientific Computing. 2. ed. Cambridge: Cambridge University Press, 1992.
SENETA, E. Non-Negative Matrices and Markov Chains. 2. ed. New York: Springer-Verlag, 1981.

SKLAR, B. Rayleigh fading channels in mobile digital communication Systems, Part I: Characterization", IEEE Communications Magazine, New York, v.35, n.9, p.136146, jul. 1997.

ULUKUS, S; YATES, R. D. Stochastic Power Control for Cellular Radio Systems. IEEE Transactions on Communications, New York, v.46, n.6, p.784-798, jun. 1998.

UYKAN, Z.; KOIVO, H. Sigmoid-Basis Nonlinear PowerControl Algorithm for Mobile Radio Systems, IEEE Transactions on Vehicular Technology., v.53, n.1, p. 265271, jan. 2004.

VERHULST, P. F. (1838). Notice sur la loi que la population pursuit dans son accroissement. Corresp. Math. Phys. v.10, p.113-121 